\title{
Application of Wireless Sensor Network Technology in Security Control of Intelligent Buildings
}

\author{
https://doi.org/10.3991/ijoe.v14i05.8652 \\ Jin Wang, Hua Shao $\left({ }^{\square}\right)$ \\ Yancheng Institute of Technology, Yancheng, China \\ 466185057 @qq.com
}

\begin{abstract}
When a wireless sensor network is used to perform real-time security monitoring inside a building, there are drawbacks like multi-path signal fading and difficulty in spectrum sensing. In light of these problems, this paper proposes an improved signal spectrum sensing algorithm based on support vector machine (SVM), which inhibits the impacts brought by the low signal-noiseratio (SNR) environment in the transmission process of wireless sensor signals through the embedded cyclostationary characteristic parameters. Based on this, considering the low efficiency and poor fault tolerance of multi-task monitoring and scheduling inside the building, this paper also proposes a multi-task coordination and scheduling algorithm based on physical information integration, which achieves multi-task scheduling and execution through intelligent breakdown and prioritization of general tasks. The simulation test shows that, compared with the artificial neural network (ANN) algorithm and the maximumminimum eigenvalue (MME) algorithm, the proposed algorithm has much better spectrum sensing effect under low SNR, takes less computation time, and achieves higher accuracy in large-scale multi-task coordination and scheduling. The research conclusions can provide new ideas for the application of wireless sensor network in intelligent building security monitoring.
\end{abstract}

Keywords—-wireless sensor network; intelligent building; spectrum sensing; multi-task coordination; security monitoring

\section{$1 \quad$ Introduction}

In recent years, fire, flood, gas explosion and indoor environmental pollution have frequently occurred in all kinds of buildings. Therefore, it is of great significance to establishing and improving real-time monitoring systems for buildings to realize unattended and intelligent building security control [1-3].

A building security monitoring system mainly consists of a sensor cluster network, a data processing centre and an information management system, but the traditional building security monitoring system cannot well achieve continuous monitoring and accurate analysis of accidents [4-7]. Intelligent building security control based on wireless sensor network (WSN) is a newly proposed method for building monitoring [8-10]. WSN has such merits as low wiring cost, continuous monitoring, unattended 
and remote control, but it also has deficiencies like electromagnetic interference, indistinguishable environmental noise, and inability of sensors to be embedded in buildings [11-13].

When a wireless sensor network is used to perform monitoring inside a building, there are drawbacks like multi-path signal fading and difficulty in spectrum sensing [14-16]. To address these problems, researchers proposed signal classification and energy detection algorithms such as the maximum-minimum eigenvalue method, artificial neural network algorithm and SVM method, but these methods all have shortcomings like poor detection effect, low sensing accuracy [17-18]; regarding the low WSN-based multi-task monitoring and scheduling efficiency and poor fault tolerance, etc., researchers proposed minimum time algorithm, genetic algorithm and minimum execution time/earliest finish time algorithm, etc. [19-21], but as the multi-task coordination inside buildings is a problem integrating data and computational task, the above methods can easily result in local node overload, low search efficiency, and low task assignment success rate in the calculation process [22-25].

In light of the above problems, this paper proposes an improved signal spectrum sensing algorithm based on SVM, which inhibits the impacts brought by the low SNR environment in the transmission process of wireless sensor signals through the embedded cyclostationary characteristic parameters. Based on this, considering the low efficiency and poor fault tolerance of multi-task monitoring and scheduling inside the building, this paper also proposes a multi-task coordination and scheduling algorithm based on physical information integration, which achieves multi-task scheduling and execution through intelligent breakdown and prioritization of general tasks. The research conclusions can provide new ideas for the application of wireless sensor network in intelligent building security monitoring.

\section{Design of the intelligent building WSN system}

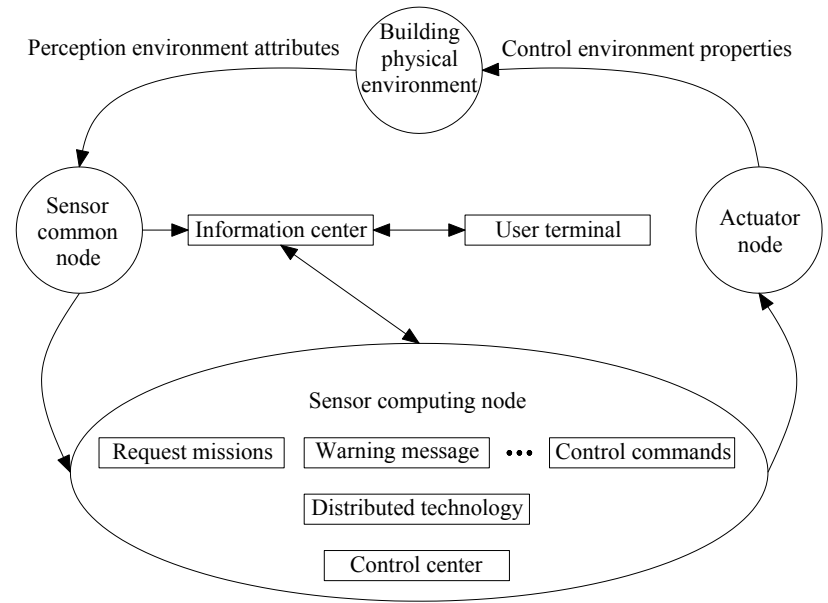

Fig. 1. Design of the intelligent building WSN system 
The design of the intelligent building WSN system is shown in Fig.1. It is mainly composed of sensor computing nodes, internal physical environment of the building, an information centre and a user terminal. The WSN monitors the internal environment of the building in real time and feeds back the attributes of the environment through common nodes to the information centre. The processing commands of the information centre are transmitted back to the internal environment of the building through the actuator node.

\section{WSN-based indoor spectrum sensing algorithm}

\subsection{Indoor spectrum sensing model}

Take one building for an example. Suppose that there are $\mathrm{W}$ primary users and $\mathrm{M}$ secondary users in the building network set, the network system can be expressed as follows:

$$
\left\{\begin{array}{l}
H_{0}: y(t)=n(t) \\
H_{1}: y(t)=\sum_{w=1}^{W} s_{w}(t)+n(t)
\end{array}\right.
$$

$\mathrm{H}_{0} / \mathrm{H}_{1}$ indicates that the system has a/no main user; $\mathrm{T}$ is the overall sampling time; and $s_{\mathrm{w}}(\mathrm{t})$ is zero mean signal, whose autocorrelation function and cyclic spectrum are:

$$
\begin{aligned}
& R^{a}(\tau)=\frac{1}{T_{0}} \int_{0}^{T_{0}} R(t, \tau) \exp (-2 \pi a t j) \mathrm{d} t \\
& S(f)=\frac{1}{N T} \sum_{n=1}^{N} Y\left(t_{n}, f+a / 2\right) Y^{*}\left(t_{n}, f-a / 2\right)
\end{aligned}
$$

$a$ is the cyclic frequency; and $\mathrm{Y}\left(\mathrm{t}_{\mathrm{n}}, f\right)$ is the Fourier transform. The identification of the signal spectrum mainly takes the maximum spectral correlation coefficient $\mathrm{M}_{\mathrm{c}}$, the spectral average energy $\mathrm{E}$, and the spectral function $\mathrm{M}_{\mathrm{s}}$ when $a=1 / \mathrm{T}_{0}$ as input vectors. The expression is as follows:

$$
\begin{aligned}
& \left\{\begin{array}{l}
C_{y}^{a}(f)=S^{a}(f) /[S(f+a / 2) S(f-a / 2)]^{1 / 2} \\
M_{C}=\max \left|C_{y}^{a}(f)\right|
\end{array}\right. \\
& M_{S}=\left.S^{a}(f)\right|_{a=1 / T_{0}}
\end{aligned}
$$

The spectrum sensing input vector is $\mathrm{X}=\left\{\begin{array}{ll}\mathrm{E} & \mathrm{M}_{\mathrm{c}} \mathrm{M}_{\mathrm{s}}\end{array}\right\}$. It is used as the training sample to train the SVM. After the training, the monitoring signals collected from the 
site are used as actual detection samples for spectrum sensing at different wireless sensor nodes. The decision function for different spectrum sensing is expressed as follows:

$$
f(x)=\operatorname{sgn}\left\{\sum_{i=1}^{l}\left(a_{i} y_{i} K\left(x_{i}, x\right)+b_{i}\right)\right\}
$$

\subsection{Simulation and verification}

The algorithm proposed in this paper was compared with the MME algorithm and the ANN algorithm to verify its spectrum sensing performance inside the building. The simulation software was Matlab 2012b, and 3 kinds of modulated signals AM (single frequency sine wave), BPSK and MSK were set, of which the latter two are of random signal sequences. Different SNRs were set for calculation.

Table 1 shows the spectrum sensing accuracy of the MME algorithm, the ANN algorithm, and the proposed algorithm with respect to the three kinds of modulated signals at a SNR of $-12 \mathrm{~dB}$ and $0 \mathrm{~dB}$. From the table, it can be seen that when the SNR decreased, the spectrum sensing accuracy of the MME algorithm and the ANN algorithm decreased significantly, while the proposed algorithm achieved better sensing accuracy at both SNRs. When the SNR was $-12 \mathrm{~dB}$, the accuracy of the proposed algorithm was higher than those of the MME algorithm and the ANN algorithm by $70.7 \%$ and $22.7 \%$.

Table 1. Spectrum sensing accuracy of the three algorithms at a SNR of $-12 \mathrm{~dB}$ and $0 \mathrm{~dB}$

\begin{tabular}{|l|c|c|c|c|c|c|}
\hline \multirow{2}{*}{$\begin{array}{c}\text { Modulation } \\
\text { type }\end{array}$} & \multicolumn{3}{|c|}{$\boldsymbol{P}_{\mathrm{c}}\left(\boldsymbol{R}_{\mathrm{sn}}=-\mathbf{1 2 d B}\right)$} & \multicolumn{3}{c|}{$\boldsymbol{P}_{\mathrm{c}}\left(\boldsymbol{R}_{\mathrm{sn}}=\mathbf{0 d B}\right)$} \\
\cline { 2 - 7 } & $\boldsymbol{M M E}$ & $\boldsymbol{A N N}$ & Proposed & $\boldsymbol{M M E}$ & $\boldsymbol{A N N}$ & Proposed \\
\hline AM & 17.9 & 70.2 & 81.5 & 84.6 & 87.3 & 91.1 \\
\hline BPSK & 11.8 & 64.6 & 77.8 & 79.0 & 83.7 & 86.8 \\
\hline MSK & 8.7 & 56.7 & 79.4 & 76.8 & 82.9 & 89.4 \\
\hline
\end{tabular}

Fig.2 illustrates the detection accuracy of the three algorithms under different SNR conditions with respect to two modulated signals - AM (the upper one) and BPSK (the lower one). As can be seen, when the SNR was greater than $0 \mathrm{~dB}$, the spectrum sensing accuracy of the 3 algorithms was all high, but when the SNR was less than $5 \mathrm{~dB}$, the accuracy of the MME algorithm drastically decreased, and that of the ANN algorithm also decreased greatly, while the proposed algorithm could maintain an accuracy of over $75 \%$. 


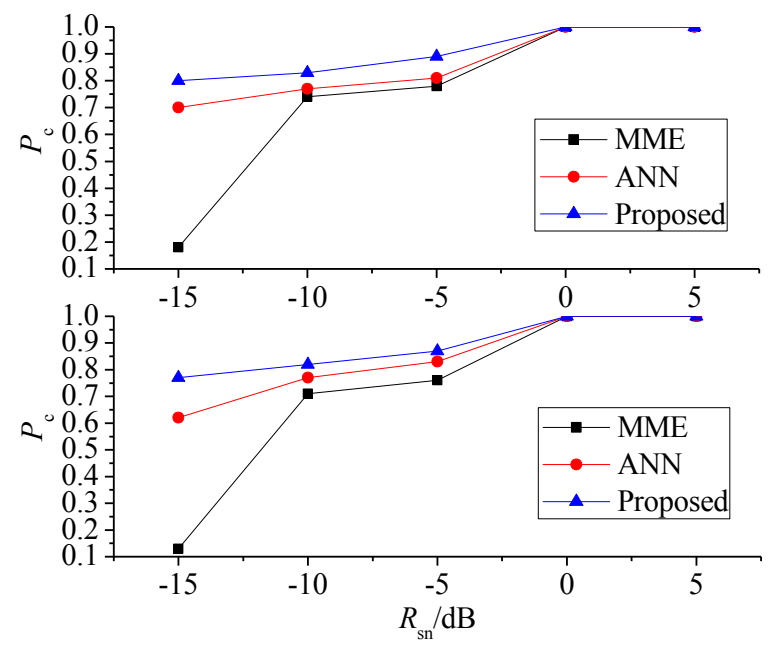

Fig. 2. Detection accuracy of the 3 algorithms at different SNRs with respect to AM (the upper one) and BPSK (the lower one) modulated signals

\section{WSN-based indoor multi-monitoring-task scheduling optimization}

\subsection{Multi-monitoring-task scheduling algorithm}

Fig.3 shows the structure of the indoor multi-monitoring-task scheduling in an intelligent building. The structure mainly addresses the task allocation and task scheduling. For a specific task, the system decomposes it into multiple subtasks by calculating the correlation constraint relationships and computational complexity, etc., and assigns the subtasks to different computing nodes one by one. The task scheduler performs big data analysis to determine in what sequence these subtasks will be executed.



Fig. 3. Multi-monitoring-task scheduling structure 
This paper carries out intelligent distribution of the multi-task WSN monitoring based on the multi-band Turing machine model. Let there be a total of $\mathrm{N}$ working bands, the relational system be $\mathrm{M}$, the initial state of the Turing machine be $\mathrm{q}_{0}$, the input task be $\mathrm{w}$, and the time when $\mathrm{M}$ accepts $\mathrm{w}$ be $\mathrm{t}(\mathrm{w})$. The Turing machine server exists inside each monitoring node in the whole wireless sensor system. Based on the complexity of the monitoring task, the server divides it into multiple subtasks superimposed. The Turing machine server achieves optimal breakdown and allocation of the monitoring task according to the complexity of the task and sorts the sub-tasks according to the calculated degree of difficulty. If a node cannot complete the task after computing for a period of time, it will break down and match the sub-task for a second time until the overall task is completed.

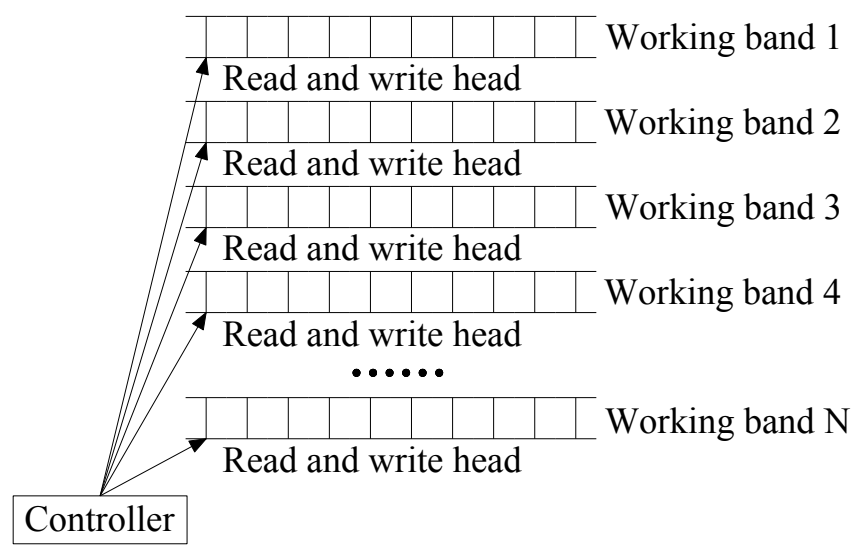

Fig. 4. Multi-belt Turing machine model

According to the above analysis, a WSN-based indoor dynamic multi-monitoringtask scheduling algorithm is established, as shown in Fig.5. The proposed scheduling algorithm targets the shortest overall multi-task execution time and the highest task execution efficiency. Prior to multi-task scheduling, the first step is to query the directed acyclic graph parameters of related tasks, randomly generate a schedule list, and calculate the priority of different tasks according to Equation (7).

$$
B L\left(t_{i}\right)=\frac{w\left(t_{i}\right)}{M_{p}}+\max _{t_{j} \in \operatorname{succ}\left(t_{i}\right)}\left(\frac{C\left(t_{i}, t_{j}\right)}{M_{c}}+B L\left(t_{j}\right)\right)
$$

$\mathrm{M}_{\mathrm{p}}$ and $\mathrm{M}_{\mathrm{c}}$ are the median values of unit calculation and link transmission capacities, respectively. 


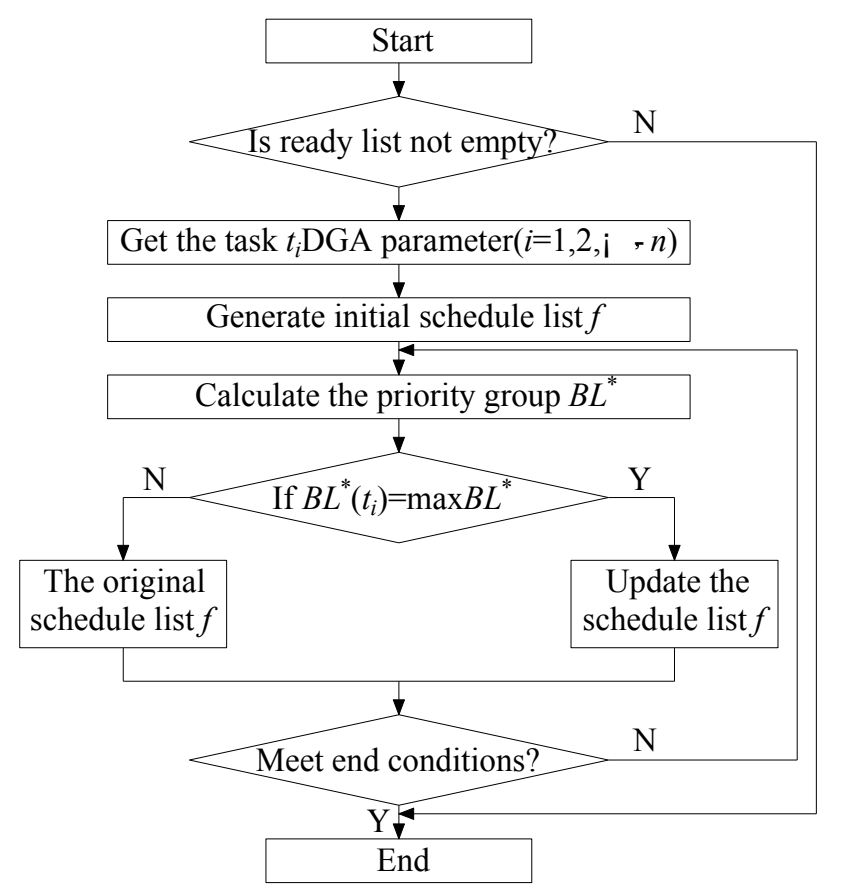

Fig. 5. WSN-based indoor dynamic multi-monitoring-task scheduling algorithm

\subsection{Test results and analysis}

The simulation software was Matlab 2012b. 20 common sensor nodes were arranged inside a building, and 30-150 tasks were generated randomly. Fig.6 shows the overall task scheduling time of the MM algorithm, the genetic algorithm, the MCT algorithm and the proposed algorithm with different numbers of tasks. As can be seen from the figure, when the number of tasks increased, the scheduling time of all the algorithms tended to increase. When the number of tasks reached 100, the task scheduling time of the proposed algorithm is $265 \mathrm{~ms}$, much lower than those of the MM algorithm $(299 \mathrm{~ms})$, genetic algorithm(308ms) and MCT algorithm(330ms). Compared with the other three algorithms, the proposed algorithm is improved in terms of node load balancing, resource allocation, and prevention of premature convergence.

Fig.7 shows the histogram of the overall task scheduling time when the number of tasks was $30,50,60,100$, and 150 . As can be seen, the proposed algorithm took the minimum time to complete all five numbers of tasks. This was because the proposed scheduling algorithm broke down the task with high complexity into several sub-tasks with lower complexity, and used the sorting mechanism to assign them to the corresponding computing nodes according to the degree of difficulty. It can also be seen that as the number of tasks increased, the computational advantage of the proposed algorithm became more obvious. 


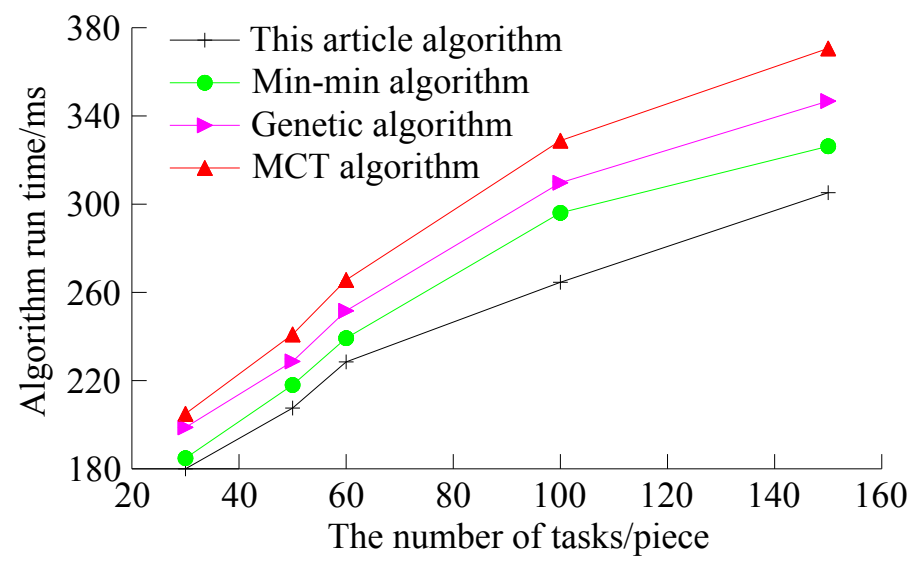

Fig. 6. Computation time of different algorithms

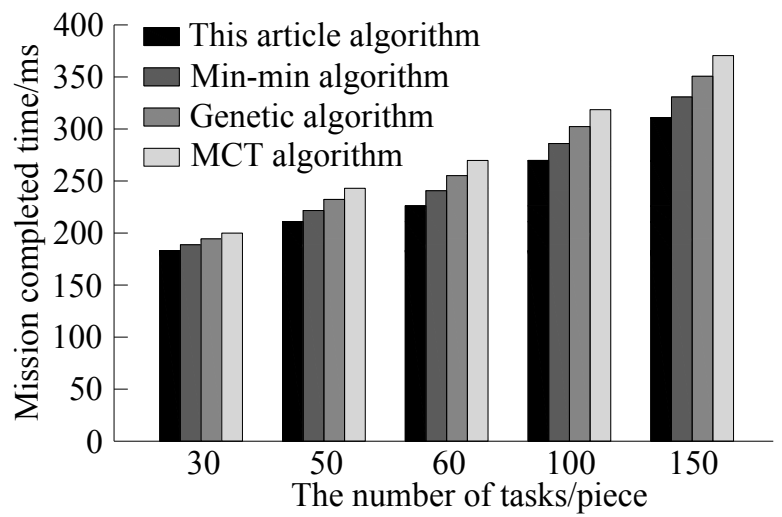

Fig. 7. Time taken by the 4 algorithms to complete different number of tasks

Fig. 8 shows the curve relationship between the number of task and the task scheduling success rate under each of the four algorithms. As can be seen, as the number of tasks increased, the task scheduling success rates of the four algorithms all tended to decrease. When the number of tasks reached 150, the task scheduling success rate of the MCT algorithm decreased the most, which was generally $76 \%$, followed by that of the genetic algorithm, which was $82 \%$, and then it was the success rate of the MM algorithm, which was $87 \%$. The task scheduling success rate of the proposed algorithm reached $90 \%$. This was because the other three algorithms prioritized the tasks that were less time consuming, but in this way the overall scheduling of tasks was often not optimal. 


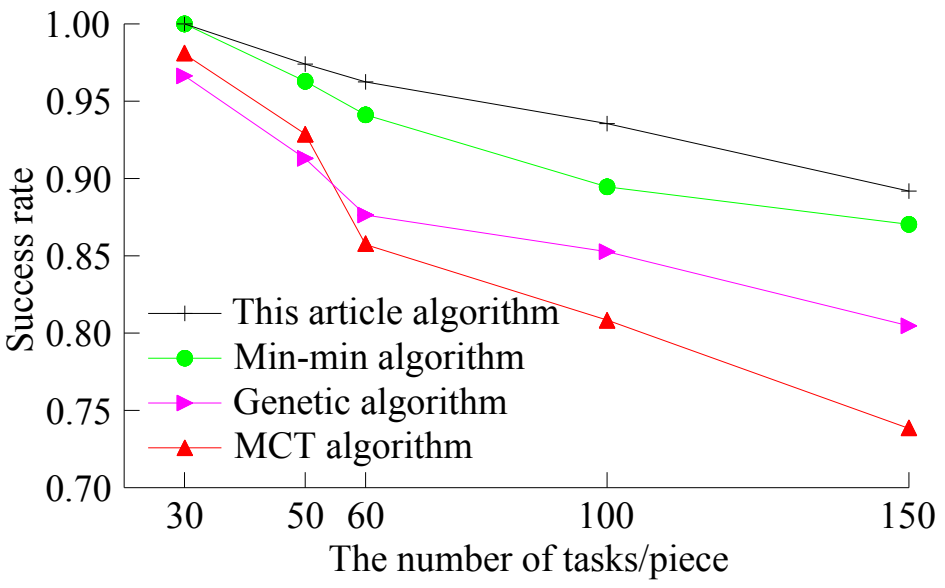

Fig. 8. Relation curve between number of tasks and task scheduling success rate

\section{Conclusions}

When a wireless sensor network is used to perform real-time security monitoring inside a building, there are drawbacks like multi-path signal fading and difficulty in spectrum sensing. In light of these problems, this paper proposes an improved signal spectrum sensing algorithm based on support vector machine (SVM), which inhibits the impacts brought by the low signal-noise-ratio (SNR) environment in the transmission process of wireless sensor signals through the embedded cyclostationary characteristic parameters. Based on this, considering the low efficiency and poor fault tolerance of multi-task monitoring and scheduling inside the building, this paper also proposes a multi-task coordination and scheduling algorithm based on physical information integration. Through simulation test, this paper proves the feasibility of this algorithm. The conclusions are as follows:

1. The WSN-based indoor spectrum sensing algorithm proposed can still have a high spectrum reconfiguration ability when the SNR is low, and when the SNR is reduced to $-15 \mathrm{~dB}$, the spectrum sensing accuracy rate can still be over $75 \%$.

2. The multi-task scheduling algorithm for indoor building monitoring proposed in this paper has improvements in node load balancing, resource allocation and prevention of premature convergence. It decomposes the task with high overall complexity into several sub-tasks with low complexity and uses the sorting mechanism to assign the sub-tasks to the corresponding computing nodes according to the degree of difficulty. For large-scale multi-task coordination and scheduling, it has higher accuracy. 


\section{Acknowledgement}

(1) Jiangsu Province Industry, Universities, Research Institutions Forward-Looking Joint Research Projects. Research on Passive Energy Saving Technology for the Enclosure Products of Assembled Residential Buildings in Jiangsu Coastal Area (No.BY2016065-50);

(2) Ministry of Housing and Urban Rural Development of Science and Technology of Projects in People's Republic of China. Research on Integration Technology of Energy Saving Renovation of Existing Residential Buildings in Jiangsu Coastal Area. (No.2014-K1-050).

\section{$7 \quad$ References}

[1] Cannistraro, G., Cannistraro, M., Cannistraro, A., Galvagno A. (2016). Analysis of air pollution in the urban center of four cities Sicilian, International Journal of Heat and Technology, 34(S2), S219-S225. https://doi.org/10.18280/ijht.34Sp0205

[2] Wang, W., Wang, N., Jafer, E., Hayes, M., O'Flynn, B., O'Mathuna, C. (2010). Autonomous wireless sensor network based Building Energy and Environment Monitoring system design. International Conference on Environmental Science and Information Application Technology, 3, 367-372, IEEE. https://doi.org/10.1109/ESIAT.2010.5568311

[3] Gattuso, D., Greco, A., Marino, C., Nucara, A., Pietrafesa, M., Scopelliti, F. (2016). Sustainable mobility: environmental and economic analysis of a cable railway, powered by photovoltaic system, International Journal of Heat and Technology, 34(1), 7-14. https://doi.org/10.18280/ijht.340102

[4] Ou, J., Li, H. (2003). Wireless sensor information fusion for structural health monitoring. Proceedings of SPIE - The International Society for Optical Engineering, 5099, 356-362. https://doi.org/10.1117/12.487067

[5] Qiu, W.M., Guo, D.W., Zhang, C.Y., Xu, Y.X., Wang, G. (2015). A smart grid client-side testing platform for monitoring, Mathematical Modelling of Engineering Problems, 2(3), 17-20. https://doi.org/10.18280/mmep.020305

[6] Zerbes, M.V., Șitoianu, C.I. (2015). Improving food radioactivity monitoring process in the laboratory of the nuclear radiation hygiene from sibiu public health department, Academic Journal of Manufacturing Engineering, 13(2), 84-89.

[7] Boubrima, A., Matigot, F., Bechkit, W., Rivano, H., Ruas, A. (2015). Optimal deployment of wireless sensor networks for air pollution monitoring. 1-7. https://doi.org/10.1109/IC CCN.2015.7288443

[8] Wang, S.C., Yan, K.Q., Ho, C.L., Wang, S.S. (2014). The optimal generalized byzantine agreement in cluster-based wireless sensor networks. Computer Standards \& Interfaces, 36(5), 821-830. https://doi.org/10.1016/j.csi.2014.01.005

[9] Wang, S. S., Wang, S. C., Yan, K. Q. (2014). Reaching trusted byzantine agreement in a cluster-based wireless sensor network. Wireless Personal Communications, 78(2), 10791094. https://doi.org/10.1007/s11277-014-1802-3

[10] Wang, S.C., Yan, K.Q., Ho, C. L., Wang, S.S. (2013). Reaching agreement in a dual fallible cluster-based wireless sensor network. Applied Mechanics \& Materials, 284-287, 2152-2156. https://doi.org/10.4028/www.scientific.net/AMM.284-287.2152 
[11] Li, H.M., Liu, H.H. (2017). The optimizated study on preparation process of nano tire pressure sensor used in auto, Academic Journal of Manufacturing Engineering, 15(1), 97104.

[12] Tang, R.X., Zhang Z.J., Liu Y.L., Wang Z.P. (2017). Spatial information is exploited to ad-just hand movement when hitting moving target, NeuroQuantology, 15(3), 7-11.

[13] Mathúna, C. Ó., O’Donnell, T., Martinez-Catala, R. V., Rohan, J., O’Flynn, B. (2008). Energy scavenging for long-term deployable wireless sensor networks. Talanta, 75(3), 613.. https://doi.org/10.1016/j.talanta.2007.12.021

[14] Chatterjea, S., Nieberg, T., Meratnia, N., \& Havinga, P. (2008). A distributed and selforganizing scheduling algorithm for energy-efficient data aggregation in wireless sensor networks. Acm Transactions on Sensor Networks, 4(4), 1-41. https://doi.org/10.1145/138 7663.1387666

[15] Toumpis, S., Tassiulas, L. (2006). Optimal deployment of large wireless sensor networks. IEEE Transactions on Information Theory, 52(7), 2935-2953. https://doi.org/10.1109/ TIT.2006.876256

[16] Pacheco-Paramo, D., Pla, V., Martinez-Bauset, J. (2009). Cognitive radio networks. Vehicular Technology Magazine IEEE, 4(4), 76-84. https://doi.org/10.1002/9780470742 $\underline{020 . c h 6}$

[17] Gomes, R. D., Adissi, M. O., Lima-Filho, A. C., Spohn, M. A., Belo, F. A. (2013). On the impact of local processing for motor monitoring systems in industrial environments using wireless sensor networks. International Journal of Distributed Sensor Networks, (2013-716), 2013(1), 276-277. https://doi.org/10.1155/2013/471917

[18] Abdaoui, A., El-Fouly, T.M. (2014). Tossim and distributed binary consensus algorithm in wireless sensor networks. Journal of Network \& Computer Applications, 41, 451-458. https://doi.org/10.1016/j.jnca.2014.01.010

[19] Tian, J., Gao, M., Ge, G. (2016). Wireless sensor network node optimal coverage based on improved genetic algorithm and binary ant colony algorithm. Eurasip Journal on Wireless Communications \& Networking, (1), 104. https://doi.org/10.1186/s13638-016-0605-5

[20] Sun, K., Ning, P., Wang, C. (2006). Tinysersync: secure and resilient time synchronization in wireless sensor networks. IEEE Journal on Selected Areas in Communications, 24(2), 395-408. https://doi.org/10.1007/978-0-387-46276-9 15

[21] Stelte, B. (2012). Unidirectional traffic flow to protect wireless sensor networks against byzantine attacks. Communications in Computer \& Information Science, 314, 503-518. https://doi.org/10.1007/978-3-642-35755-8 36

[22] Fan, Y.M., Qin, B.T. (2010). Genetic algorithm for multiple sink node optimal deployment in large scale wireless sensor networks. Transducer \& Microsystem Technologies, 29(6), 32-35.

[23] Banimelhem, O., Mowafi, M., Aljoby, W. (2013). Genetic algorithm based node deployment in hybrid wireless sensor networks. Communications \& Network, 5(4), 273-279. https://doi.org/10.4236/cn.2013.54034

[24] Sarangi, S., Kar, S. (2011). Genetic algorithm based mobility aware clustering for energy efficient routing in wireless sensor networks, 354(1), 1-6. https://doi.org/10.1109/ICON. 2011.6168497

[25] Li, M., Shi, W. R. (2012). Optimal sensor deployment scheme for heterogeneous wireless sensor networks. Journal of Chongqing University, 35(2), 55-59. 


\section{Authors}

Jin Wang: (1978.09.09).Associate professor of Yancheng Institute of Technology. Dean of the Department of Architecture. His research direction is architectural intelligence and green building.

Hua Shao: (1991.07.21). Hua Shao is a joint master at Yancheng Institute of Technology and China University of Mining and Technology. Her research direction is landscape planning and design. Her interest is in landscape design and architecture intelligentization.

Article submitted 30 March 2018. Final acceptance 05 May 2018. Final version published as submitted by the authors. 